\title{
SOME BAIRE SPACES FOR WHICH BLUMBERG'S THEOREM DOES NOT HOLD
}

\author{
H. E. WHITE, JR.
}

ABSTRACT. First, in the second section, we describe a class of Baire spaces for which Blumberg's theorem does not hold. Then, in the third section, we discuss Blumberg's theorem for $P$-spaces.

1. In [2], J. C. Bradford and C. Goffman proved that a metrizable space $X$ is a Baire space if and only if the following statement, called Blumberg's theorem, holds.

1.1 If $f$ is a real valued function defined on $X$, then there is a dense subset $D$ of $X$ such that $f \mid D$ is continuous.

It follows from their proof that every topological space for which 1.1 holds is a Baire space. In [15], the author gave several examples of completely regular, Hausdorff, Baire spaces for which, if $2^{\boldsymbol{N}_{0}}=\boldsymbol{\kappa}_{1}, 1.1$ does not hold (see also [13], [14]). In $\$ 2$ we establish, using a lemm a from [14], a result which shows that there are a number of Baire spaces for which 1.1 does not hold.

2. For any topological space $X$, we denote the weight of $X$, the pseudoweight of $X$, the density character of $X$, and the ring of all bounded real valued, continuous functions defined on $X$ by $w X, \pi w X, \delta X$, and $C^{*}(X)$, respectively (see $[4, \mathrm{p} .619])$. For any subset $A$ of $X$, we denote the closure of $A$ by $\mathrm{cl} A$. We denote the set of all real numbers by $R$.

2.1 Theorem. Suppose $X$ is a Baire space of cardinality $2^{\boldsymbol{N}_{0}}$ such that

(a) $X$ satisfies the countable chain condition,

(b) $w X=\delta X=2^{\boldsymbol{N}_{0}}$, and

(c) every set of the first category in $X$ is nowhere dense in $X$.

Then 1.1 does not hold for $X$.

Proof. Let $\mathcal{B}$ denote a base for the topology $\mathcal{T}$ on $X$ of cardinality $2^{\boldsymbol{N}_{0}}$ such that $\varnothing, X \in \mathfrak{B}$. We may assume that $\mathfrak{B}$ is closed under countable union.

Received by the editors August 30,1973 and, in revised form, May 17, 1974.

AMS (MOS) subject clas sifications (1970). Primary 54C30, 54F 99, 54G20。

Key words and phrases. Baire space, Blumberg's theorem. 
Let $M$ denote the set of all real valued functions defined on $X$ that are measurable $(\mathcal{S})$, where $\mathcal{S}$ is the $\sigma$-algebra generated by $\mathcal{B}$. We shall now prove the following statement.

2.2 Suppose $f: X \rightarrow R$ and there is a dense subset $D$ of $X$ such that $f \mid D$ is continuous. Then there is $g$ in $M$ such that $\{x \in X: f(x)=g(x)\}$ is dense in $X$.

Because (a) holds, there is a function $\gamma: \mathcal{T} \rightarrow \mathcal{B}$ such that for each $U$ in $\mathcal{T}, \gamma(U)$ is a dense subset of $U$. By Lemma 1.1 of [14] (see also [6, p. 202], there is a $G_{\delta}$ set $K$ containing $D$ and a continuous, real valued function $h$ defined on $K$ such that $h|D=f| D$. Let $\left\{V_{n}: n \in N\right\}$ (N denotes the set of natural numbers) denote a base for the usual topology on $R$. For each $n$ in $N$, choose $U_{n}$ in $\mathcal{T}$ such that $h^{-1}\left[V_{n}\right]=U_{n} \cap K$. Suppose $K=\bigcap\left\{G_{n}\right.$ : $n \in N\}$, where each $G_{n}$ is open. Let $C$ denote the union of $\bigcup\left\{X \sim \gamma\left(G_{n}\right)\right.$ : $n \in N\}$ and $\bigcup\left\{U_{n} \sim \gamma\left(U_{n}\right): n \in N\right\}$, and let $W=\gamma(X \sim \mathrm{cl} C)$. Then, because (c) holds, $W$ is dense in $X$. It is easily checked that $W \subset K$ and, for each $n$ in $N,(h \mid W)^{-1}\left[V_{n}\right]=\gamma\left(U_{n}\right) \cap W$. So, if we define $g$ by letting

$$
g(x)= \begin{cases}h(x) & \text { if } x \in W, \\ 0 & \text { if } x \in X \sim W,\end{cases}
$$

then $g \in \mathbb{M}$. And the set $\{x \in X: f(x)=g(x)\}$ is dense in $X$ because it contains $D \cap W$.

Because $|\mathcal{B}|=2^{K_{0}}$, the cardinality of $\mathcal{S}$ is $2^{K_{0}}[9$, p. 26, exercise 9]. Now if $h \in \mathbb{M}$, then there is a sequence $\left(h_{n}\right)_{n \in N}$ of functions in $\mathbb{M}$ such that each $h_{n}$ has finite range and $\lim _{n \rightarrow \infty} h_{n}(x)=h(x)$ for all $x$ in $X$. Therefore $|\pi|=2^{x_{0}}$. The proof of Theorem 2.1 will be completed if we prove the following statement.

2.3 There is a function $f: X \rightarrow R$ such that if $g \in \mathbb{M}$, then $\mid\{x \in X:$ $f(x)=g(x)\} \mid<2^{K_{0}}$.

But 2.3 follows from a standard argument; see, for example, [8, p. 148].

2.4 Proposition. Suppose $X$ is a Baire space of cardinality $2^{\mathrm{X}_{0}}$ such that $\left|C^{*}(X)\right|=\delta X=2^{\kappa_{0}}$, and either $X$ is perfectly normal or extremally dis. connected [7, exercise $1 \mathrm{H}]$. Then 1.1 does not hold for $X$.

The proof of 2.4 is similar to the proof of 2.3. If $X$ is perfectly normal, then 2.2 is true when $\mathfrak{B}=\mathfrak{T}$. And, if $X$ is extremally disconnected, then 2.2 is true if $\mathbb{M}=C^{*}(X)$ and $f$ is assumed to be bounded on $X[7$, exercise $6 \mathrm{M}]$.

Remarks. (1) Proposition 2.4 is false if the hypothesis that $\left|C^{*}(X)\right|=$ $2^{X_{0}}$ is replaced by the hypothesis that $w X=2^{X_{0}}$. 
(2) Statements 2.1 and 2.5 remain true if the hypothesis that $w X=2^{\boldsymbol{N}_{0}}$ is replaced by the hypothesis that $\pi w X=2^{\boldsymbol{N}_{0}}$. However, if $X$ is regular, $\pi w X=2^{X_{0}}$, and $X$ satisfies the countable chain condition, then $w X=\left|C^{*}(X)\right|$ $=2^{X_{0}}$ (see 2.2 and 2.4 of [4]).

2.5 Proposition. Suppose $2^{\boldsymbol{N}_{0}}=\boldsymbol{N}_{1}$ and that $(X, \mathcal{T})$ is a Baire space that satisfies 2.1(a) and 2.1(b). If no nonempty element of $\mathcal{T}$ is separable, then there is a dense subspace $Y$ of $X$ of cardinality $2^{\boldsymbol{N}_{0}}$ such that

(1) $Y$ is a Baire space that satisfies (a), (b), and (c) of 2.1, and

(2) $Y$ is hereditarily Lindelöf.

Proof. Let $\mathcal{B}$ be as in the proof of 2.1 , and let $\mathcal{F}$ denote the family of all nowhere dense subsets $F$ of $X$ such that $X \sim F \in \mathbb{B}$. Because $X$ satisfies 2.1(a), if $K$ is a nowhere dense subset of $X$, then there is $F$ in $\mathcal{F}$ such that $K \subset F($ let $F=X \sim \gamma(X \sim \operatorname{cl} K)$ ).

Because $|\mathcal{F}|=2^{\boldsymbol{N}_{0}}$, we can construct, using the argument in [8, pp. 146147], a subset $Y$ of $X$ such that $B \cap Y \neq \varnothing$ for every nonempty $B$ in $B$ and $|F \cap Y| \leq \boldsymbol{x}_{0}$ for every $F$ in $\mathcal{F}$. It is clear that $Y$ is dense in $X$ and that it satisfies (a) and (b) of 2.1. Because $|Y \cap K| \leq \boldsymbol{x}_{0}$ for every nowhere dense subset $K$ of $X, Y$ satisfies (2) and a subset of $Y$ is nowhere dense in $Y$ if and only if it is countable. Hence $Y$ is a Baire space which satisfies (c) of 2.1 .

We conclude this section with some examples which illustrate the preceding results. We assume that $2^{\boldsymbol{N}_{0}}=\boldsymbol{N}_{1}$ throughout these examples.

(1) Suppose $(X, \mathcal{T})$ is a Souslin line. By this we mean that $\mathcal{T}$ is the interval topology induced by a total order on $X$, and that $(X, \mathcal{T})$ is a compact, connected space which satisfies the countable chain condition but which has no nonempty separable open subsets. It is clear that $X$ satisfies $2.1(a)$ and 2.1(b). By Lemma 11 of [10], $X$ satisfies 2.1(c). Therefore Theorem $2.1 \mathrm{im}$ plies that 1.1 does not hold for $X$. Because $X$ is perfectly normal, this follows from 2.4, too. $X$ seems to be the only known example of a first countable, completely regular, Hausdorff, Baire space for which 1.1 does not hold.

(2) Suppose $X$ is a quasi-regular [11, p. 164], $T_{1}$ Baire space of weight $2^{\boldsymbol{N}_{0}}$ which has no isolated points, and which admits a category measure [12, p. 156]. Then, by 2.1 , any dense subset of $X$ of cardinality $2^{\boldsymbol{N}_{0}}$ is a Baire space for which 1.1 does not hold.

(2a) Let $\mathcal{T}$ denote the density topology on the real line $R$. It was shown in [15] that $(R, \mathcal{T})$ is a Baire space for which 1.1 does not hold. This follows from 2.1 because $(R, \mathcal{J})$ admits a category measure. Indeed, this follows from 2.1 even if we replace the continuum hypothesis with the hypothesis that any 
subset of $R$ of cardinality $<2^{\aleph_{0}}$ has a Lebesgue measure 0 . And, if $(Y, \mathcal{U})$ is a compactification of $(R, \mathcal{J})$, then any dense subset of $Y$ of cardinality $2^{\aleph_{0}}$ is a Baire space which satisfies the hypothesis of 2.1.

(2b) Let $S$ denote the Stone space of the Boolean algebra $\mathcal{M}$, where $\mathcal{L}$ is the set of all Lebesgue measurable subsets of $[0,1]$ and $\pi$ is the subset of $\mathcal{Q}$ of sets of Lebesgue measure 0 . Then $S$ is a compact, Hausdorff space which admits a category measure [11, p. 163], and which has no isolated points. Therefore 1.1 does not hold for the Baire space $Z$ constructed in [11] such that $Z \times Z$ is of the first category. Because $S$ is extremally disconnected, this follows from 2.4, too.

(3) Let $X=R \times R$ and let $U$ denote the product topology on $X$ induced by the density topology on $R$. Then $X$ satisfies (a) and (b) of 2.1. However, it, does not satisfy 2.1 (c) because the set $D=\{(x, y) \in X: x-y$ is rational $\}$ is a dense subset of $X$ which is of the first category in $X$. Because no nonempty open subset of $X$ is separable, it follows from 2.5 that there is a dense subset $Y$ of $X$ which satisfies the hypothesis of 2.1 . Note that if $Y$ is obtained as in the proof of 2.5, then the Lebesgue measure of $Y$ is 0 . The author does not know whether 1.1 holds for $X$.

3. Obviously, 1.1 holds for every discrete space. For pseudo-discrete spaces ( $P$-spaces; see $[7$, p. 62]), the situation is more complex. As an example due to $M$. Henrikson shows, a $P$-space need not be a Baire space. And, even if the $P$-space $X$ is strongly $\alpha$-favorable [3, p. 116], 1.1 need not hold for $X$ (see [13]). However, if $2^{\boldsymbol{K}_{0}}=\boldsymbol{K}_{1}$, then 1.1 holds for every cocompact [1, p. 292] $P$-space. This follows from the next statement, the proof of which is essentially the same as the proof of 1.13 of [15].

3.1 Proposition. If $2^{\boldsymbol{x}_{0}}=\boldsymbol{x}_{1}$, then 1.1 holds for every quasi-regular, cocompact space $X$ for which every nonempty $G_{\delta}$ has nonempty interior.

Remark. A metrizable space is cocompact if and only if it is strongly $\alpha$-favorable (see Theorem 1 of [1] and Theorem 8.7 of [3]).

If $\left(\left(X_{i}, \mathcal{J}_{i}\right)\right)_{i \in I}$ is a family of topological spaces and $m$ is an infinite cardinal number, then we denote by $\mathcal{J}(m)$ the $m$-box product topology on $X=$ $\Pi\left\{X_{i}: i \in I\right\}$ induced by $\left(\mathcal{T}_{i}\right)_{i \in I^{*}}$. The following two statements guarantee a supply of cocompact $P$-spaces.

3.2 Proposition. Suppose $m$ is an infinite cardinal number such that, if $m_{n}<m$ for all $n$ in $N$, then $\Sigma\left\{m_{n}: n \in N\right\}<m$. If, for each $i$ in $I,\left(X_{i}, \mathcal{T}_{i}\right)$ is a cocompact P-space, then $(X, \mathcal{J}(\mathrm{m}))$ is a cocompact P-space. 
3.3 Proposition. If $(X, \mathcal{T})$ is a locally compact, Hausdorff space and $\mathcal{T}_{\pi}$ denotes the coarsest $P$-space topology for $X$ containing $\mathcal{T}[5, p .55]$, then $\left(X, \mathcal{J}_{\pi}\right)$ is cocompact.

Proof of 3.2. It is easily verified that $(X, \mathcal{T}(\mathrm{m}))$ is a $P$-space. And if for each $i$ in $I, \mathcal{U}_{i}$ is a compact cotopology for $\mathcal{T}_{i}$, then $\mathrm{U}\left(\boldsymbol{\aleph}_{0}\right)$ is a compact cotopology for $\mathscr{T}(\mathrm{m})$ [1, p. 242].

Proposition 3.3 is easily proven by using $3.11(\mathrm{~b})$ of [7]. In fact, if $\mathcal{T}$ is compact, then $\mathcal{T}$ is a compact cotopology for $\mathcal{T}_{\pi}$.

Proposition 3.2 provides an example of a cocompact $P$-space for which, if $2^{K_{0}}=2^{K_{1}}, 1.1$ does not hold. For let $I$ be a set of cardinality $K_{1}$ and, for each $i$ in $I$, let $\mathcal{T}_{i}$ denote the discrete topology on the two point set $X_{i}$. Then Proposition 1.2 of [14] implies that 1.1 does not hold for $\left(X, \mathcal{T}\left(\boldsymbol{x}_{1}\right)\right)$, provided $2^{\boldsymbol{K}_{0}}=2^{\boldsymbol{K}_{1}}$.

Remark. It is obvious that if 1.1 does not hold for a topological space $X$, then it does not hold for any dense subspace of $X$. However, it can happen that (a) every dense subset of $X$ of cardinality $2^{\boldsymbol{K}_{0}}$ is a Baire space for which 1.1 does not hold, and (b) 1.1 holds for $X$. For let $X=\beta N \sim N$, where $\beta N$ denotes the Stone-Čech compactification of the discrete space $N$. By Proposition 1.2 of [14], (a) holds. But, if $2^{\aleph_{0}}=\aleph_{1}$, Proposition 3.1 implies that (b) holds.

\section{REFERENCES}

1. J. M. Aarts, J. de Groot and R. H. McDowell, Cotopology for metrizable spaces, Duke Math. J. 37 (1970), 291-295. MR 41 \#488.

2. J.C. Bradford and C. Goffman, Metric spaces in which Blumberg's theorem holds, Proc. Amer. Math. Soc. 11 (1960), 667-670. MR 26 \#3832.

3. G. Choquet, Lectures on analysis. I: Integration and topological vector spaces, Benjamin, New York, 1969. MR 40 \#3252; 44-1630.

4. W. W. Comfort and Anthony W. Hager, Estimates for the number of real-valued continuous functions, Trans. Amer. Math. Soc. 150 (1970), 619-631. MR 41 \#7621.

5. W. W. Comfort and S. Negrepontis, Homeomorphs of three subspaces of $\beta N \backslash N$, Math. Z. 107 (1968), 53-58. MR 38 \#2739.

6. R. Engelking, Outline of general topology, PWN, Warsaw, 1965; English transl., North-Holland, Amsterdam; Interscience, New York, 1968. MR 36 \#4508; 37 \#5836.

7. L. Gillman and M. Jerison, Rings of continuous functions, University Ser. in Higher Math., Van Nostrand, Princeton, N. J., 1960. MR 22 \#6994.

8. C. Goffman, Real functions, Rinehart, New York, 1953. MR 14, 855.

9. P. R. Halmos, Measure theory, Van Nostrand, Princeton, N. J., 1950. MR 11, 504. 
10. J. L. Kelley, Measures on Boolean algebras, Pacific J. Math. 9 (1959), 1165-1177. MR 21 \#7286.

11. J. C. Oxtoby, Cartesian products of Baire spaces, Fund. Math. 49 (1960/61), 157-166. MR 25 \#4055; 26-1543.

12. - Spaces that admit a category measure, J. Reine Angew. Math. 205 (1960/61), pp. 156-170. MR 25 \#4054; 26-1543.

13. R. Levy, A totally ordered Baire space for which Blumberg's theorem fails, Proc. Amer. Math. Soc. 41 (1973), 304; Erratum 45 (1974), 469.

14. - Strongly non-Blumberg spaces (to appear).

15. H. E. White, Jr., Topological spaces in which Blumberg's theorem holds, Proc. Amer. Math. Soc. 44 (1974), 454-462.

671 S. EUREKA AVENUE, COLUMBUS, OHIO 43204 